\title{
Ambient Measurements of Highly Oxidized Gas-Phase \\ Molecules during the Southern Oxidant and Aerosol Study (SOAS) 2013
}

Massoli, Paola

2018-07

Massoli , P , Stark , H , Canagaratna , M R , Krechmer , J E , Xu , L , Ng , N L , Mauldin , R L , Yan , C , Kimmel , J , Misztal , P K, Jimenez , J L , Jayne , J T \& Worsnop , D R 2018 , ' Ambient Measurements of Highly Oxidized Gas-Phase Molecules during the Southern Oxidant and Aerosol Study (SOAS) 2013 ' , ACS Earth and Space Chemistry , vol. 2 , no. 7 , pp. 653-672 . https://doi.org/10.1021/acsearthspacechem.8b00028

http://hdl.handle.net/10138/308513

https://doi.org/10.1021/acsearthspacechem.8b00028

unspecified

acceptedVersion

Downloaded from Helda, University of Helsinki institutional repository.

This is an electronic reprint of the original article.

This reprint may differ from the original in pagination and typographic detail.

Please cite the original version. 
1

\section{Ambient Measurements of Highly Oxidized Gas Phase Molecules during the 3 Southern Oxidant and Aerosol Study (SOAS) 2013}

4

5

6 Paola Massoli ${ }^{1}$, Harald Stark ${ }^{1,2,3}$, Manjula R. Canagaratna ${ }^{1}$, Jordan E. Krechmer ${ }^{1,2,3}$, Lu Xu ${ }^{5 *}$, Nga L. $7 \quad \mathrm{Ng}^{4,5}$, Roy L. Mauldin III ${ }^{6}$, Chao Yan ${ }^{7}$, Joel Kimmel ${ }^{1,8}$, Pawel K. Misztal ${ }^{9,10, * *}$, Jose L. Jimenez ${ }^{2,3}$, 8 John T. Jayne ${ }^{1}$ and Douglas R. Worsnop ${ }^{1}$

9

10

11

12

${ }^{1}$ Center for Aerosol and Cloud Chemistry, Aerodyne Research Inc., Billerica, MA 01821

${ }^{2}$ Cooperative Institute for Research in Environmental Sciences, University of Colorado, Boulder, CO 80309

${ }^{3}$ Department of Chemistry, University of Colorado, Boulder, CO 80309

${ }^{4}$ School of Earth and Atmospheric Sciences, Georgia Institute of Technology, Atlanta, GA 30332

${ }^{5}$ School of Chemical and Biomolecular Engineering, Georgia Institute of Technology, Atlanta, GA 30332

${ }^{6}$ Department of Atmospheric and Oceanic Sciences, University of Colorado, Boulder, CO 80309

${ }^{7}$ Department of Physics, University of Helsinki, FI-00560, Helsinki, Finland

${ }^{8}$ Tofwerk AG, CH-3600, Thun, Switzerland

${ }^{9}$ Department of Environmental Science, Policy and Management, University of California, Berkeley, CA 94720

${ }^{10}$ Department of Civil and Environmental Engineering, University of California, Berkeley, CA 94720

*Now at the Division of Geological and Planetary Sciences, California Institute of Technology, Pasadena, CA 91125

** Now at Centre for Ecology \& Hydrology Edinburgh, Midlothian, EH26 0QB, UK 
Data obtained with the $\mathrm{NO}_{3}{ }^{-}$CIMS instrument must be corrected for any $\mathrm{m} / z$ dependence that is associated with processes such as transmission of ions from the ion chamber to the mass spectrometer, orthogonal extraction of the ions into the time-of-flight chamber, and detection of ion signal with the multichannel plate. Relative transmission values for the mass spectrometer as a function of $\mathrm{m} / \mathrm{z}$ were determined in a series of laboratory experiments following methods described in previous studies using perflourinated alkanoic acids with carbon chain lengths of $\mathrm{C}_{5}, \mathrm{C}_{7}, \mathrm{C}_{8}$, and $\mathrm{C}_{9 .}{ }^{1,2}$ These compounds form clusters with the $\mathrm{NO}_{3}{ }^{-}$reagent ion and with deprotonated parent molecules. As shown in Figure S1, the reagent ion clusters and the clusters consisting of 1-3 parent molecules produced a series of signals in the mass spectrum that span a range of $\mathrm{m} / \mathrm{z}$ (panel a). The experiments involved measurements of the reagent ion signals in the absence of the perfluorinated acid followed by time-series measurements of ion signal after injection of the fluoroacid of interest. The amounts of fluoroacid injected were high enough to lead to a significant decrease in the reagent ion signal. The analysis is based on the idea that in the absence of $\mathrm{m} / \mathrm{z}$ dependent transmission effects, the total ion current from all the detected ions should remain constant throughout the experiment. Thus, any change in total signal upon addition of the perfluorinated acids provides a measure of the change in ion transmission efficiencies at the $m / z$ 's of the perfluorinated acid ions compared to those of the reagent ions. Two approaches for deriving the relative transmission from the measured time series (panel b) were pursued. First, the 2-D matrix consisting of time series of all reagent and analyte ions was subjected to a matrix inversion algorithm to solve the system of linear equations:

$$
\text { Constant }=\mathrm{C} 1 * \mathrm{I} 1(\mathrm{t})+\mathrm{C} 2 * \mathrm{I} 2(\mathrm{t})+\ldots+\mathrm{CN} * \mathrm{IN}(\mathrm{t})
$$

Equation 1

where Constant is the constant signal across time series

$\mathrm{C} 1, \ldots \mathrm{CN}$ : fit coefficient

$\mathrm{I} 1(\mathrm{t}), \ldots \mathrm{IN}(\mathrm{t})$ : ion signal time series

The optimized coefficients $\mathrm{C} 1-\mathrm{CN}$ were converted to relative transmission values by inverting and normalizing to a specific coefficient, typically the coefficient related to the $\mathrm{NO}_{3}{ }^{-}$signal at $m / z 62$. Solutions to the system of linear equations from this method showed a large degree of variation (panel c). This is likely due to the unconstrained nature of the matrix method. A more constrained solution 
was achieved by assuming that relative transmission follows a functional dependence to $\mathrm{m} / \mathrm{z}$, which was derived from an average of the solutions from the matrix inversion:

$$
\mathrm{RT}=10^{\wedge}\left(\mathrm{k}^{*}\left(m / z-m / z_{0}\right)\right.
$$

The optimum value for $\mathrm{k}$ of $1.9 \pm 0.2$ with $\mathrm{m} / \mathrm{z}_{0}$ of 62 was derived from an iterative algorithm that optimized the sum of all ion signals to be constant, following Eq. 1. Much better convergence was achieved using this method (panel d). The relative transmission values were normalized to $\mathrm{m} / \mathrm{z} 62$ and, together with the calibration factor $C$ of $1.89 * 10^{\wedge} 10$ molecules $\mathrm{cm}^{-3}$, converted to absolute sensitivities (in ppb) for all ion signals.

\section{PMF error calculations, diagnostics, and correlation with external tracer}

The Positive Matrix Factorization (PMF) model expresses the measured $\mathrm{m} \times \mathrm{n}$ data matrix of CIMS mass spectra vs. time $(\mathrm{X})$ as a matrix product of two matrices corresponding to PMF factor time series (G) and PMF factor mass spectra (F):

\section{$\mathrm{X}=\mathrm{GF}+\mathrm{E}$}

where $\mathrm{E}$ is the $\mathrm{m} \times \mathrm{n}$ data matrix corresponding to the residuals between the measured matrix elements of $\mathrm{X}$ and the modelled matrix product of the factor time series and factor mass spectra. The factorization is achieved using non-negatively constrained weighted least squares in which the quantity $\mathrm{Q}$ is minimized. $\mathrm{Q}$ is defined as

$$
\mathrm{Q}=\sum_{i=1}^{m} \sum_{j=1}^{n}\left(\mathrm{E}_{\mathrm{ij}} / \sigma_{\mathrm{ij}}\right)^{2}
$$

where $E_{i j}$ refers to elements of the residual matrix and $\sigma_{i j}$ refers to the standard deviation of each data point in the $\mathrm{X}$ matrix $\left(\mathrm{X}_{\mathrm{ij}}\right)$. In the limit where the residuals of data points are equal to their standard deviations, each data point contributes a value of 1 to the total $\mathrm{Q}$ and the expected value of $\mathrm{Q}$, also referred to as $\mathrm{Q}_{\exp }$, is approximately equal to the number of elements of in the $\mathrm{X}$ matrix. Q/ $\mathrm{Q}_{\exp }$ is monitored as a diagnostic of the quality of the fits. Previous studies have provided a detailed 
description of how to calculate the standard deviation values $\left(\sigma_{\mathrm{ij}}\right)$ for PMF analysis of unit-mass resolution CIMS data. ${ }^{3}$ In this work, PMF analysis was performed on the high resolution dataset in order to utilize the additional chemical information in the mass spectra. Estimation of the uncertainties associated with high-resolution fitting, in which ion intensities are obtained from overlapping peaks, are complicated ${ }^{4}$, and methods for approximating them have not yet been fully developed. Thus $\sigma_{\mathrm{ij}}$ values are estimated according to Poisson counting statistics, the dominant source of noise in the unitmass resolution data $^{3}$, as follows:

1

$$
\sigma_{\mathrm{ij}}=\left(\mathrm{I} / \mathrm{t}_{\mathrm{s}}\right)^{0.5}
$$

Where I is the raw ion signal in ions/s, obtained before corrections for ion transmission and extraction are applied, and $t_{s}$ is the integration time in seconds. We note that the $\sigma_{i j}$ values estimated using Poisson statistics provide a lower limit for the real noise in the high-resolution data. The PMF analysis in this work utilized the PMF Evaluation Tool (PET) ${ }^{5}$ together with the PMF2 algorithm. ${ }^{6}$ The signalto-noise ratio (SNR) for each data point was calculated according to previous studies" and "bad" variables with SNR $<0.2$ were down-weighted by 10 while "weak" variables with SNR between 0.2 and 2 were down-weighted by 3. Figure S4 shows the key diagnostic plots for the Positive Matrix Factorization (PMF) performed on the SOAS $2013 \mathrm{NO}_{3}{ }^{-} \mathrm{CIMS}$ dataset. The Q/Qexp is shown as a function of the number of factors $\mathrm{P}$ (top panel, left). A 6-factor solution $(\mathrm{P}=6)$, yielding a $\mathrm{Q} / \mathrm{Q} \exp$ of 1.1 was chosen to explain this dataset. The top right panel shows the total residuals for the 6-factor solution, where the bottom panels show the distribution of Q/Qexp as a function of $m / z$ and time. The mass spectra (MS) of the 6-factors as output by PMF are shown in Figure S4 as well. It is useful to note that although the total Q/Qexp for the 6-factor solution is close to the "ideal" value of 1, the distribution of Q/Qexp shows large variability. This likely reflects the fact that the PMF assumption of constant factor profiles is not strictly followed for the species studied here due to temporal variations in the distribution of gas phase species with various ambient parameters including oxidation conditions, temperature, and relative humidity. The trend in Q/Qexp with factor number can still provide useful qualitative information for selection of the optimum number of factors. PMF factor exploration showed that the addition of factors beyond 6 did not decrease the residuals or Q/Qexp significantly, meaning that most of the data variability can be explained by these six factors (see Figure S5 for source allocation from 2 to 10 factors). 

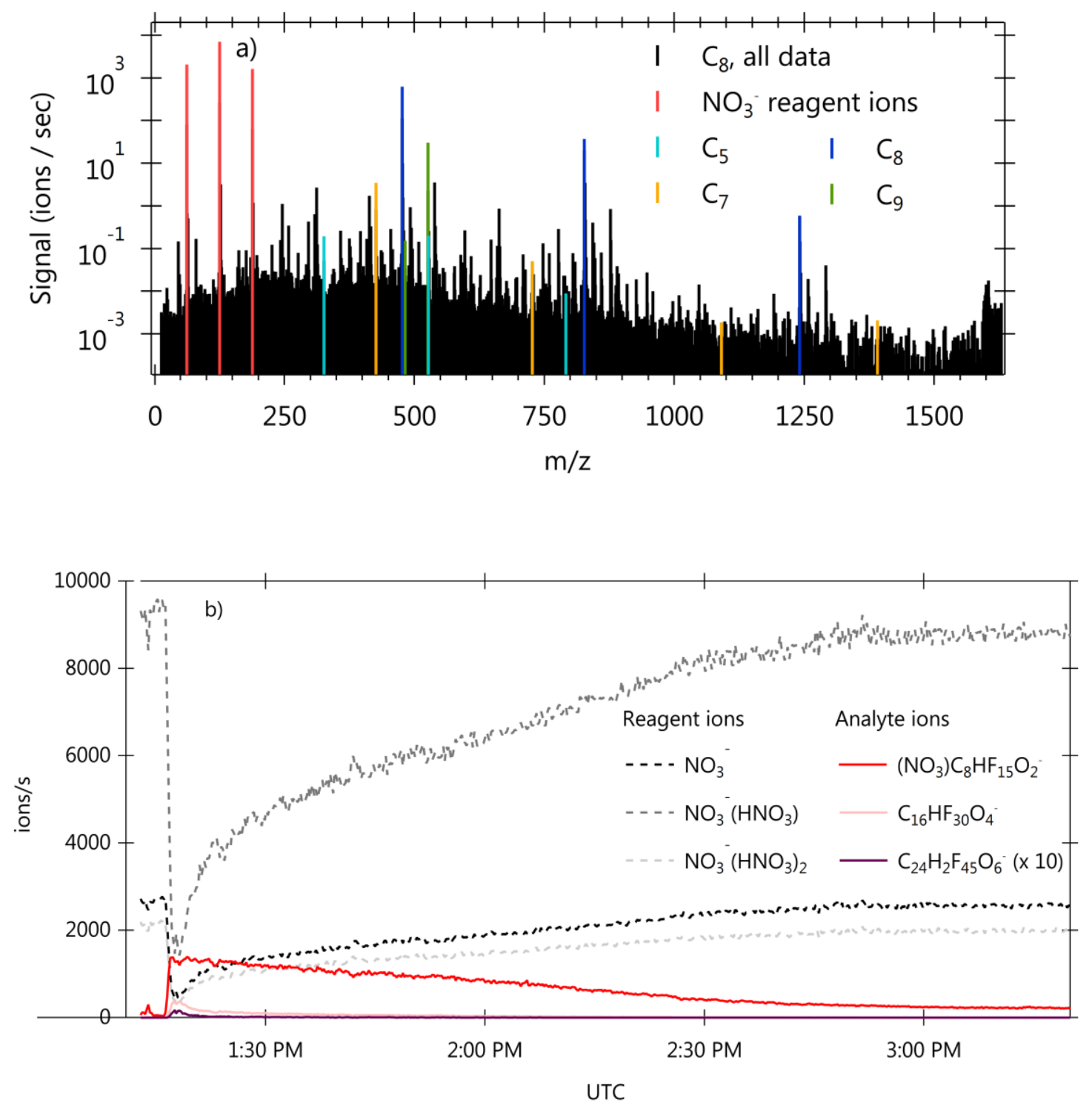

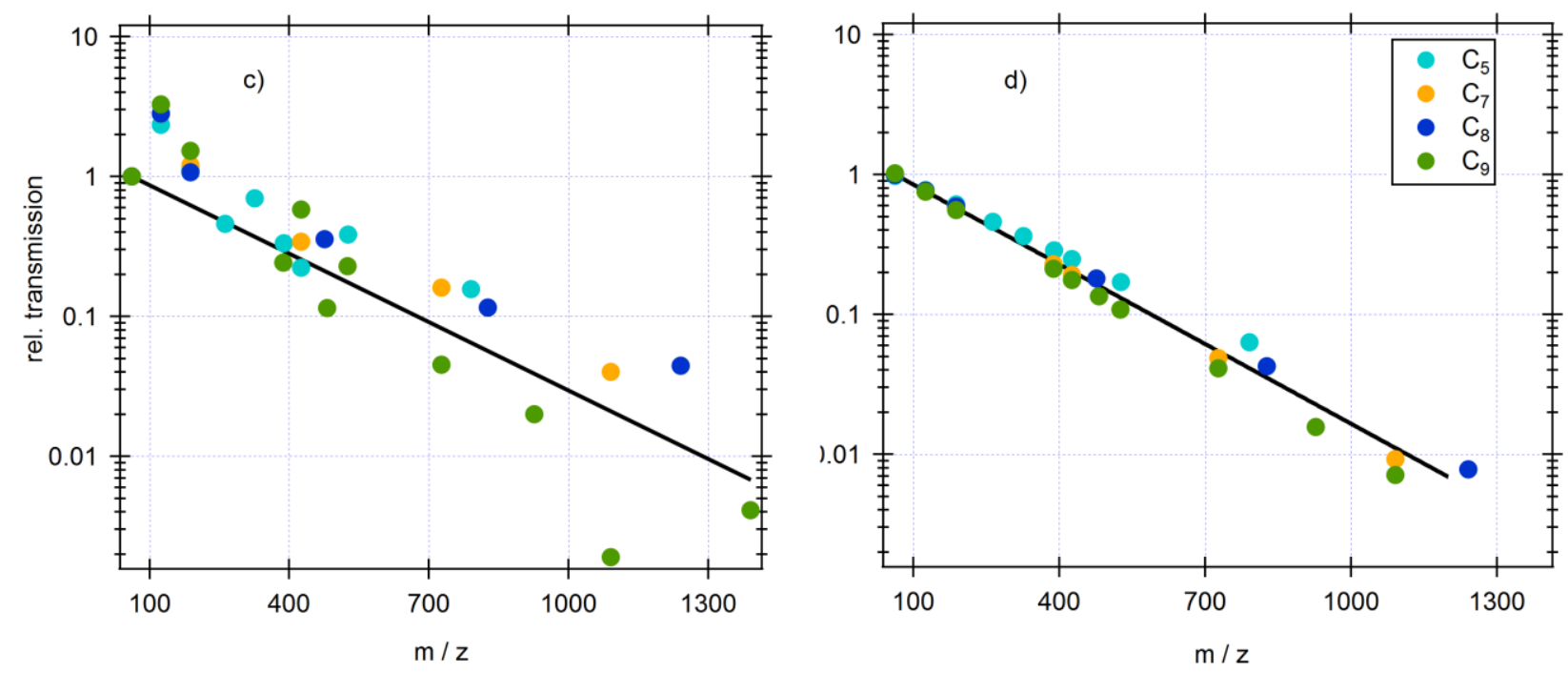

117

118 Figure S1: Results of laboratory experiments conducted to obtain transmission corrections for the $\mathrm{NO}_{3}{ }^{-}$

119 CIMS data. Panel a): mass spectrum showing ion signals from perfluorinated acids; clusters and 120 signals from deprotonated acids are shown. Panel b): time series for reagent ions and ions related to 121 perfluoroheptanoic acid $\left(\mathrm{C}_{7} \mathrm{HF}_{13} \mathrm{O}_{2}\right)$ during transmission experiments. Panels c) and d): relative 122 transmission values from matrix inversion and functional dependence methods, respectively. 


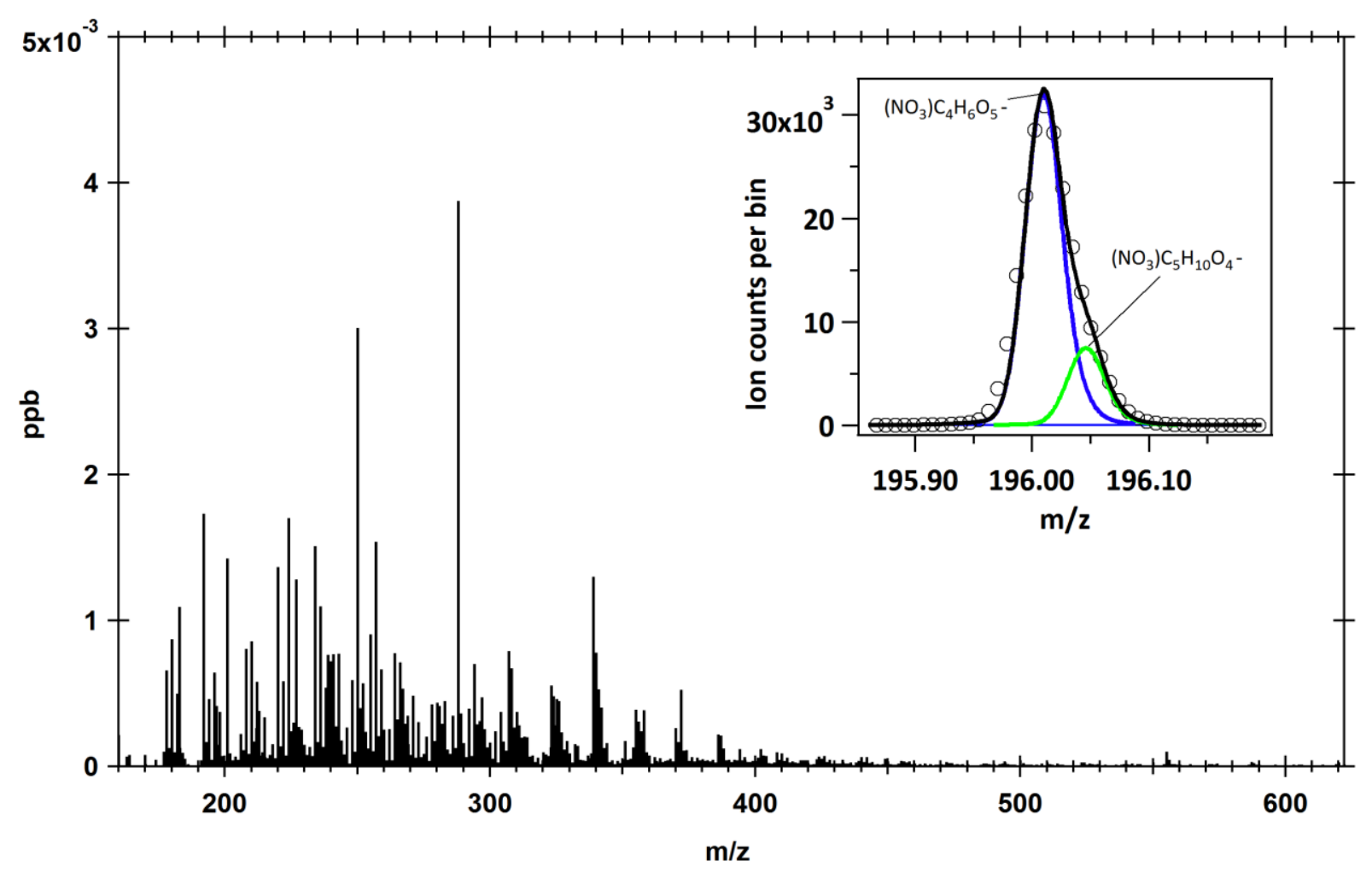

127 Figure S2: Campaign average high resolution mass spectrum with an example of molecular separation 128 using the Tofware capabilities. The mass spectral data are corrected from instrument transmission. 


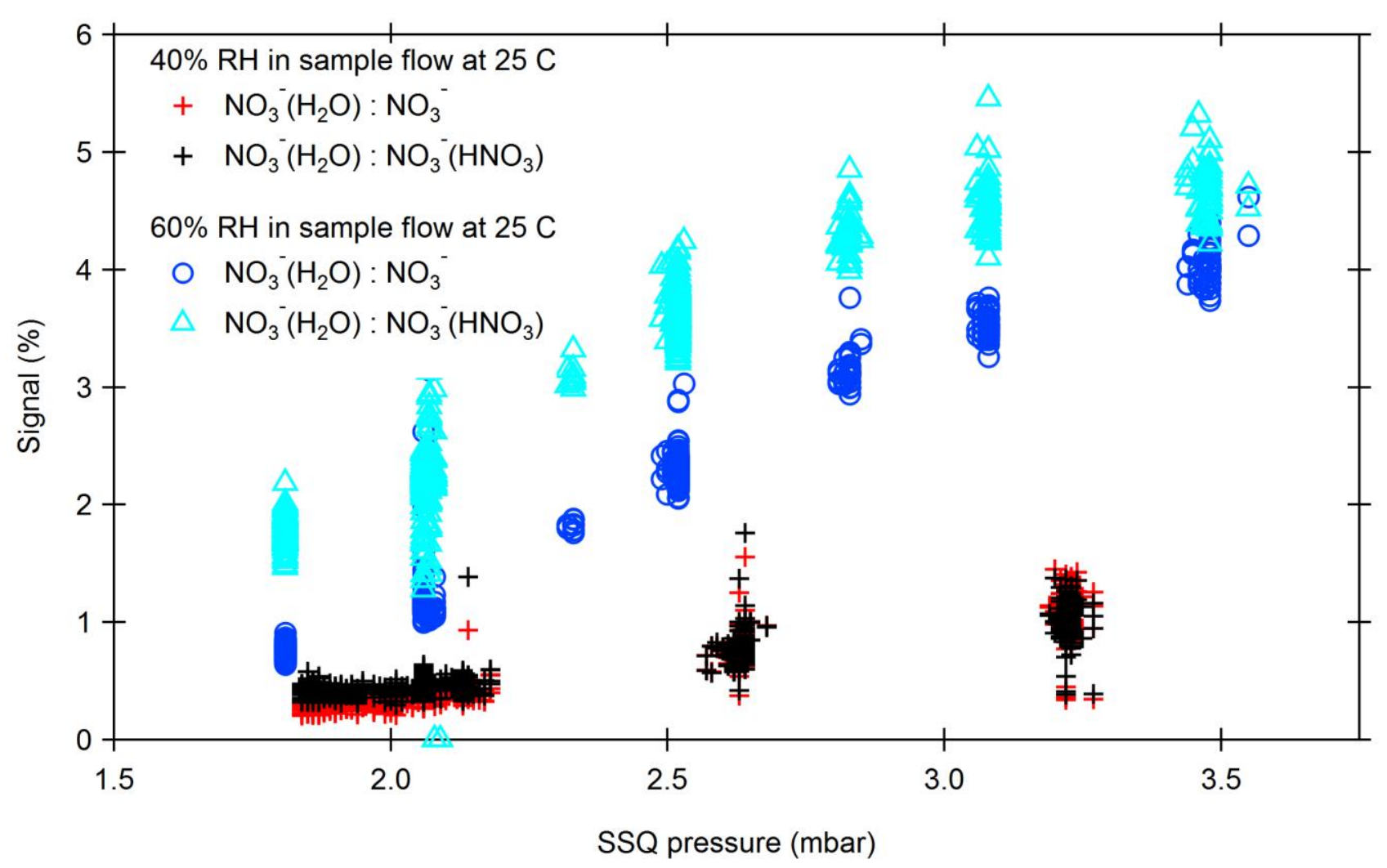

133 Figure S3: Percentage change of the ratio between the water cluster $\mathrm{NO}_{3}{ }^{-}\left(\mathrm{H}_{2} \mathrm{O}\right), \mathrm{m} / z$, 80 , and the nitrate 134 reagent ions $\mathrm{NO}_{3}{ }^{-}, \mathrm{m} / \mathrm{z}, 62$, and $\mathrm{NO}_{3}{ }^{-}\left(\mathrm{HNO}_{3}\right), \mathrm{m} / \mathrm{z}, 125$, with respect to the pressure in the first-stage 135 small quadrupole (SSQ). The ratio depends on SSQ pressure and changes faster at higher RH. 

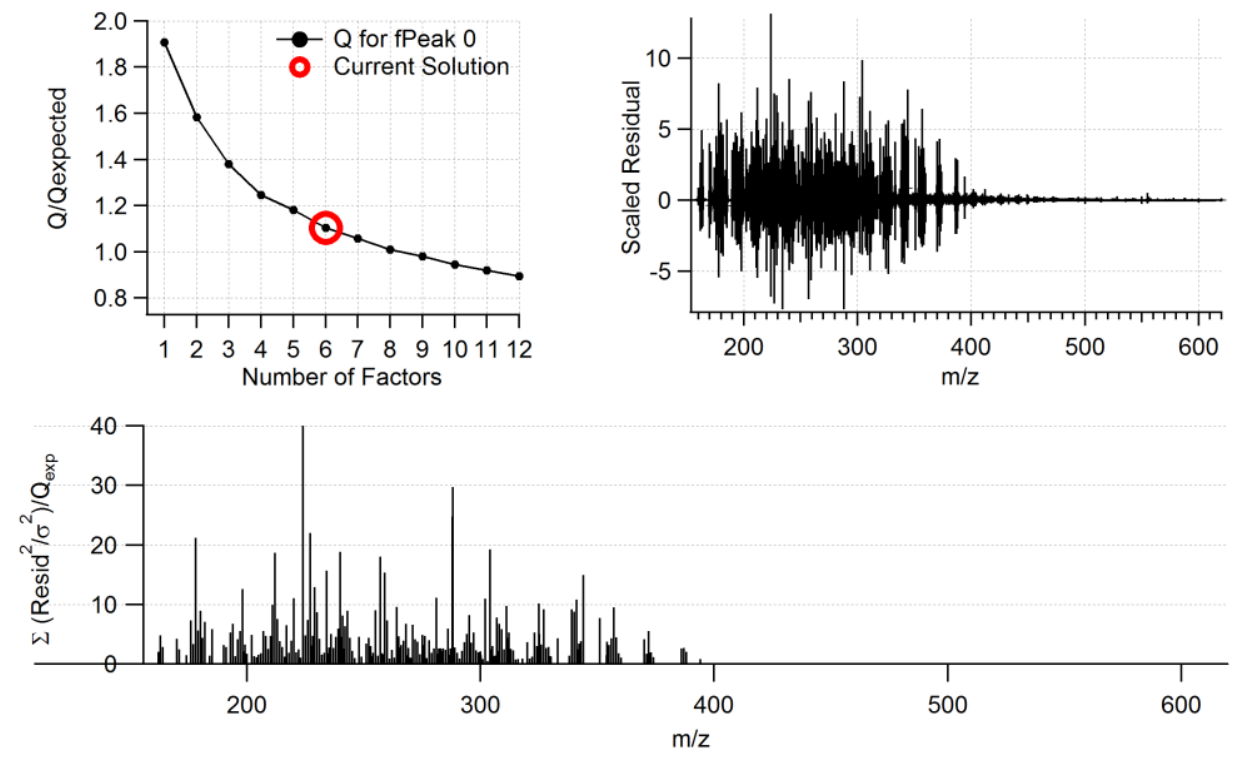

145

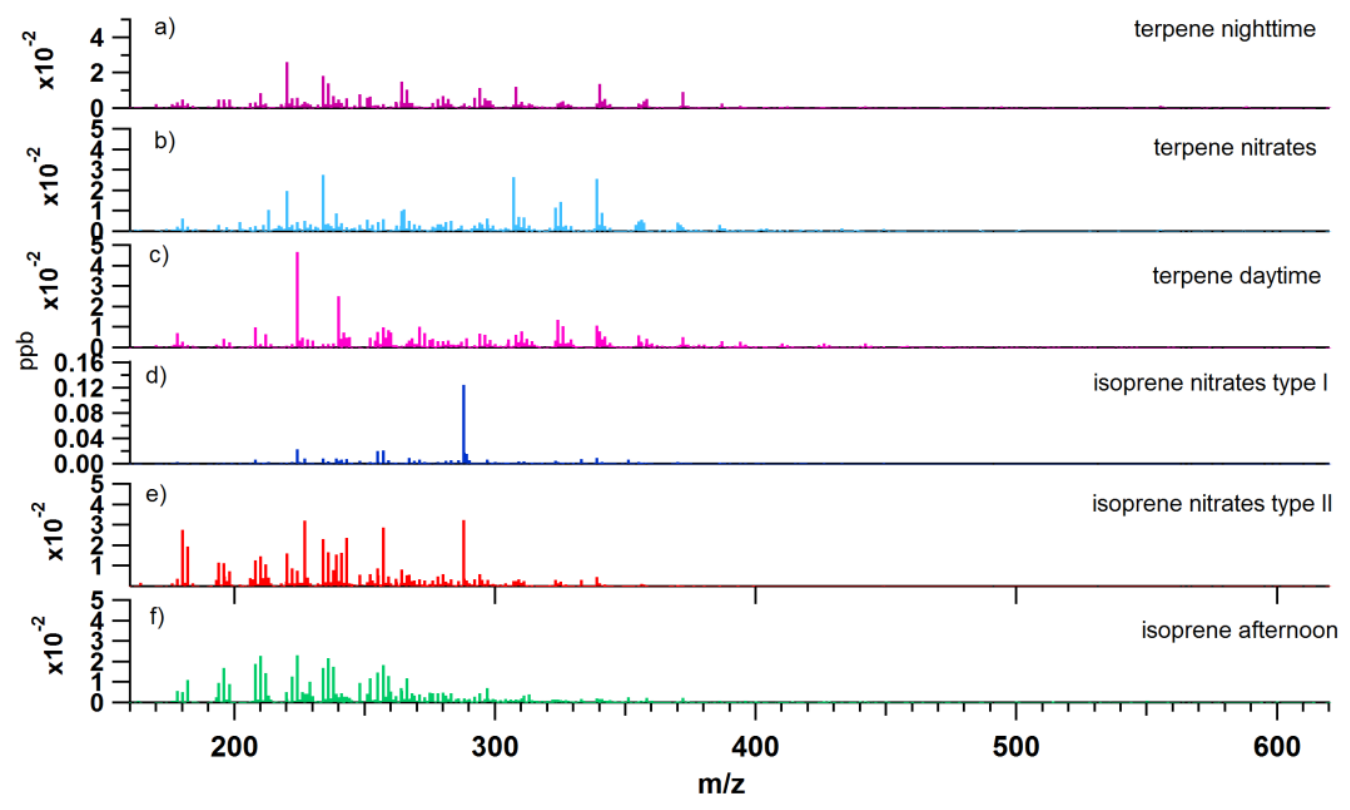

146 Figure S4: Upper panel: Summary of key diagnostic plots $\left(\mathrm{Q} / \mathrm{Q}_{\mathrm{exp}}\right.$, scaled residuals and scaled 147 residuals over $\mathrm{Q}_{\text {exp }}$ for each $\mathrm{m} / z$ ) for the PMF analysis performed on the $\mathrm{NO}_{3}{ }^{-} \mathrm{CIMS}^{-}$data collected 148 during SOAS 2013. A 6-factor solution yielding Q/Q $\mathrm{Qxp}_{2}=1.1$ was chosen. Lower panel: Mass spectra 149 (MS) of the $6 \mathrm{PMF}$ factors before splitting the MS into $\mathrm{C}_{\mathrm{n}}$ families, labeled a) to f) from top to bottom. 150 Panels a) to c) are the MS for the terpene-related factors, and panels d) to f) are the MS for the 151 isoprene-related factors. 


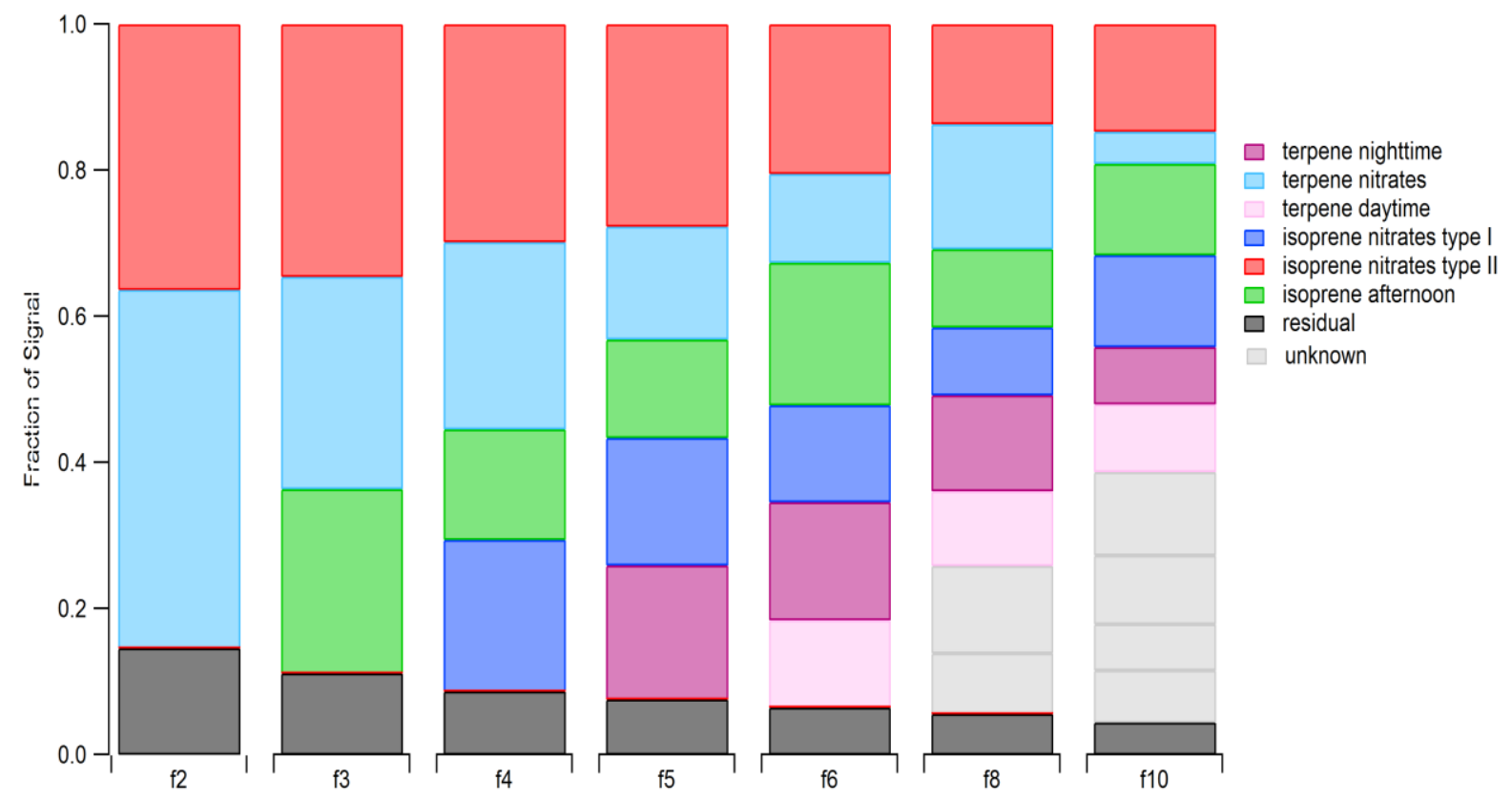

153

154 Figure S5: Source allocation from 2-10 factors PMF solution. We chose the 6-factor solution for this 155 dataset because it gave the best combination of number of factors that could be explained by chemical 156 processes and low residual. The grey sections indicated the additional factors that split at higher 157 solutions. Residuals are shown in black. 

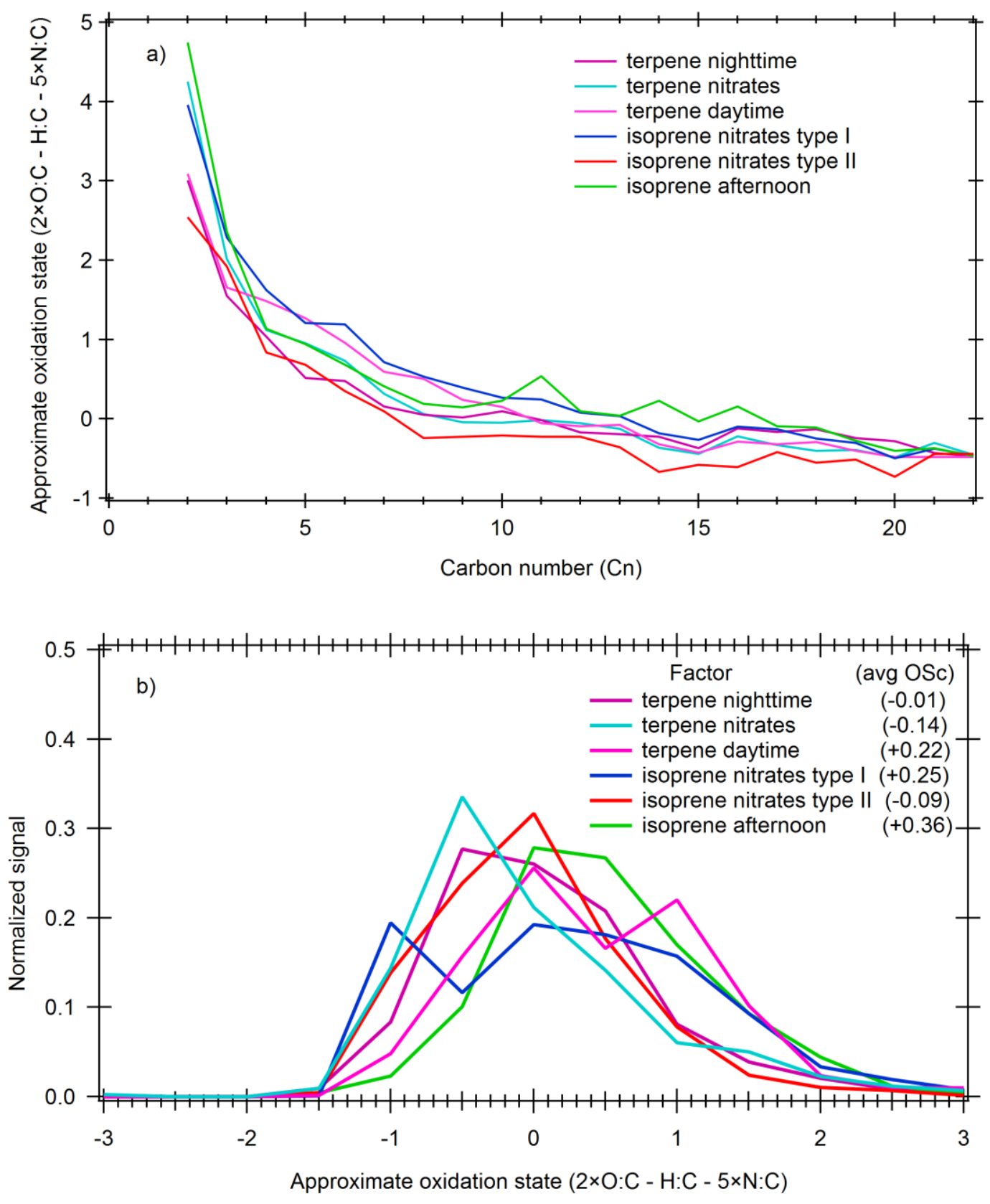

165 Figure S6: Panel a) shows the approximate oxidation state (OSc) for each factor as a function of the 166 carbon number $\mathrm{Cn}$, while panel b) shows the normalized factor signal as a function of OSc. The 167 average OSc for each factor is also reported in the legend. 


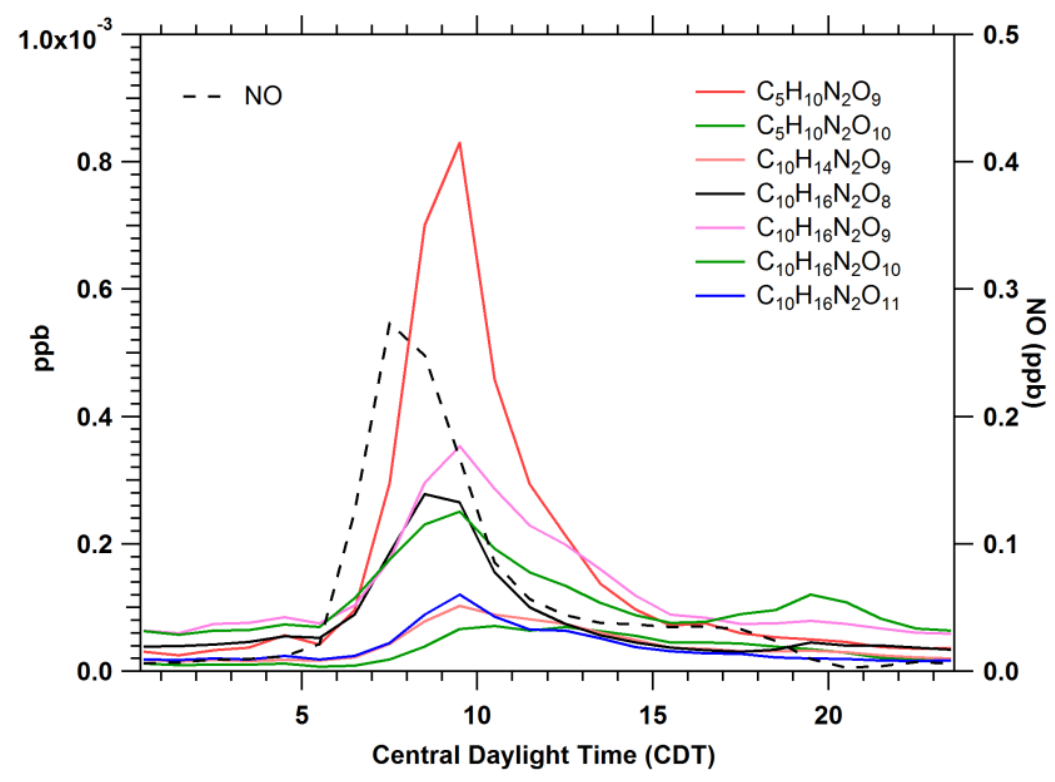

172
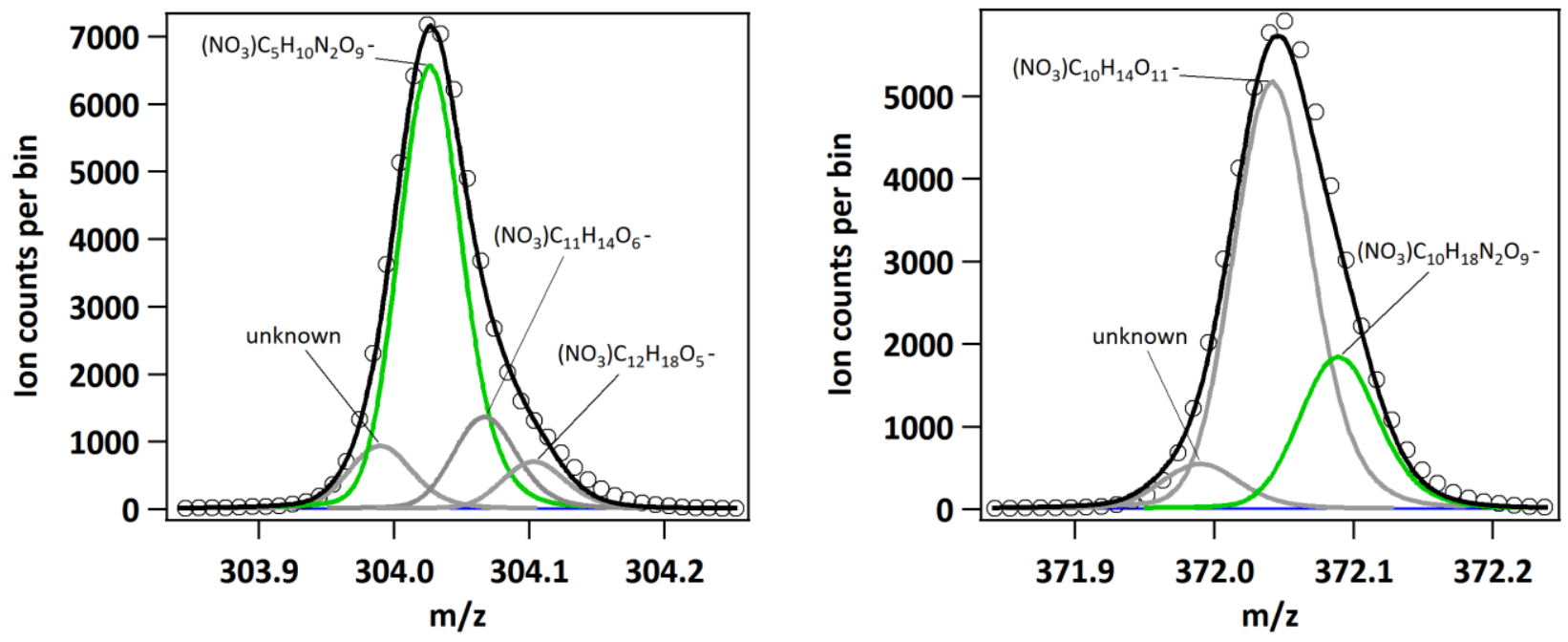

173

174 Figure S7: Top panel: diurnal cycles of $\mathrm{C}_{5}$ isoprene dinitrate and $\mathrm{C}_{10}$ monoterpene dinitrate ions. All 175 dinitrate ions peak at $\sim 1000$ CDT, approximately 2 hours after the morning NO peak. The clustering 176 reagent ion $\mathrm{NO}_{3}{ }^{-}$was omitted from the formulas to make the labels more readable. Bottom panel: high 177 resolution fits of $\mathrm{m} / \mathrm{z}, 304$ and $\mathrm{m} / \mathrm{z}, 372$, where a $\mathrm{C}_{5}$ dinitrate $\left(\mathrm{C}_{5} \mathrm{H}_{10} \mathrm{~N}_{2} \mathrm{O}_{9}\right.$, ) and a $\mathrm{C}_{10}$ dinitrate $178 \quad\left(\mathrm{C}_{10} \mathrm{H}_{18} \mathrm{~N}_{2} \mathrm{O}_{9}\right)$ are respectively detected. 
June 25, 16:00

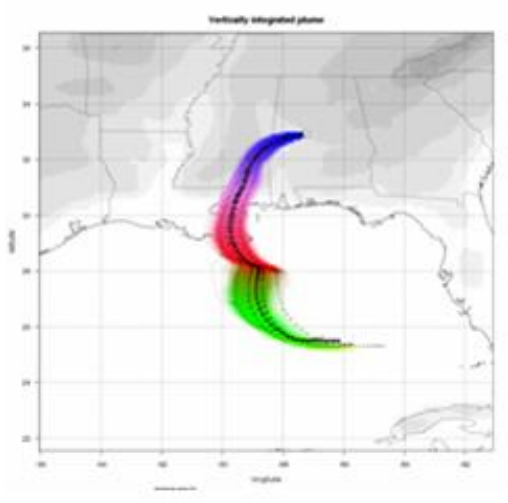

aimass age (h)

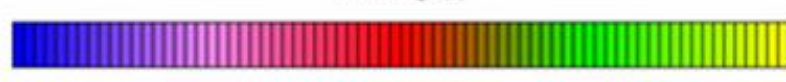

$\begin{array}{lllllllllllllllll}0 & 3 & 6 & 9 & 13 & 18 & 22 & 27 & 32 & 37 & 42 & 47 & 52 & 57 & 61 & 66 & 71\end{array}$
June 26, 18:00

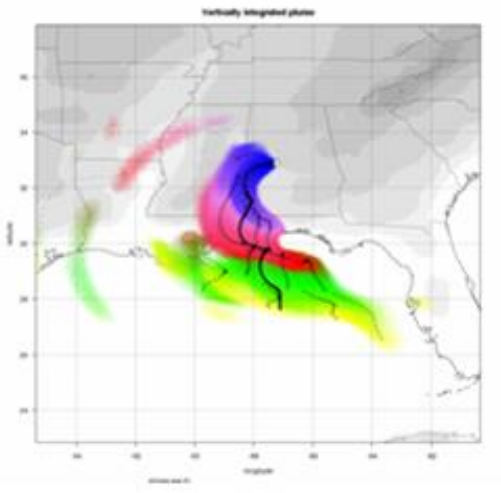

- mass-weighted center trajectory

cluster centroid (ecaled by fraction of particles)

181

182

183 Figure S8: FLEXPART back-trajectories of the air masses reaching the CTR site during June 25-26 184 and July 3, when high levels of $\mathrm{SO}_{2}$ were observed. These air masses most likely carried emissions 185 from coal fired power plants to the site. 


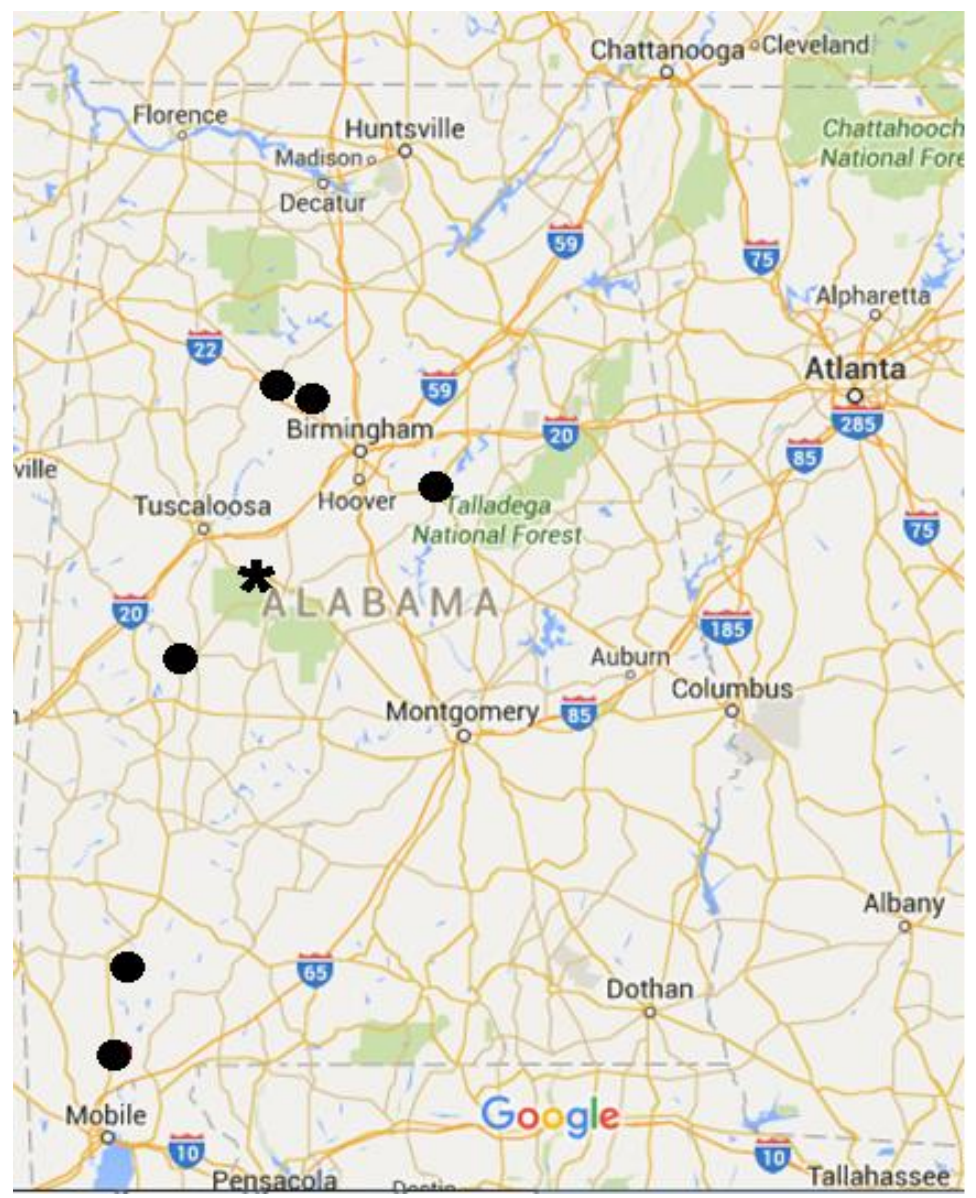

197

198 Figure S9: Map of Alabama with major coal-fired power plants (black circles) and location of 199 Centreville, where the CTR and SEARCH sites were located (black star)

200

201

202

203 

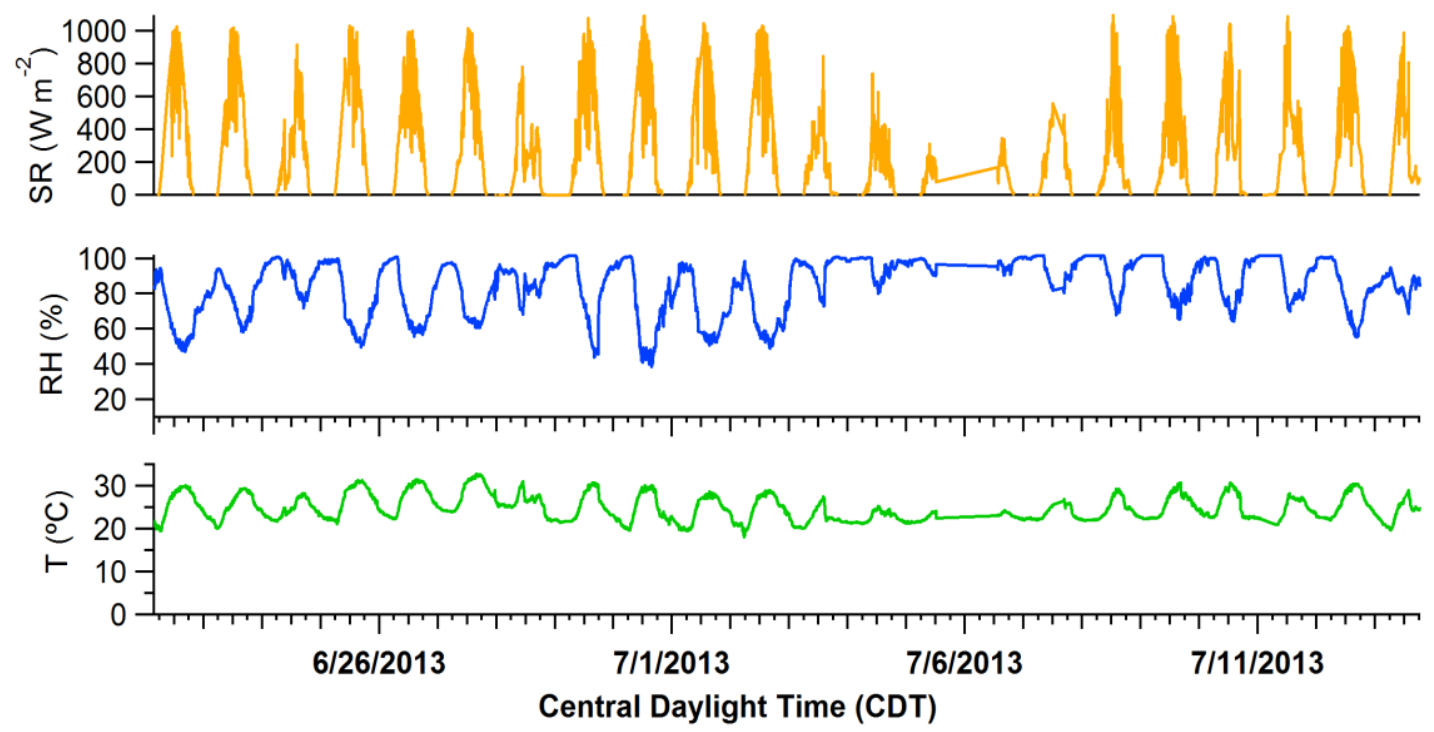

206

207 Figure S10: Temporal series of temperature (T), relative humidity (RH) and solar radiation (SR) at the 208 CTR site during SOAS 2013.

209

210

211

212

213

214

215

216

217

218 


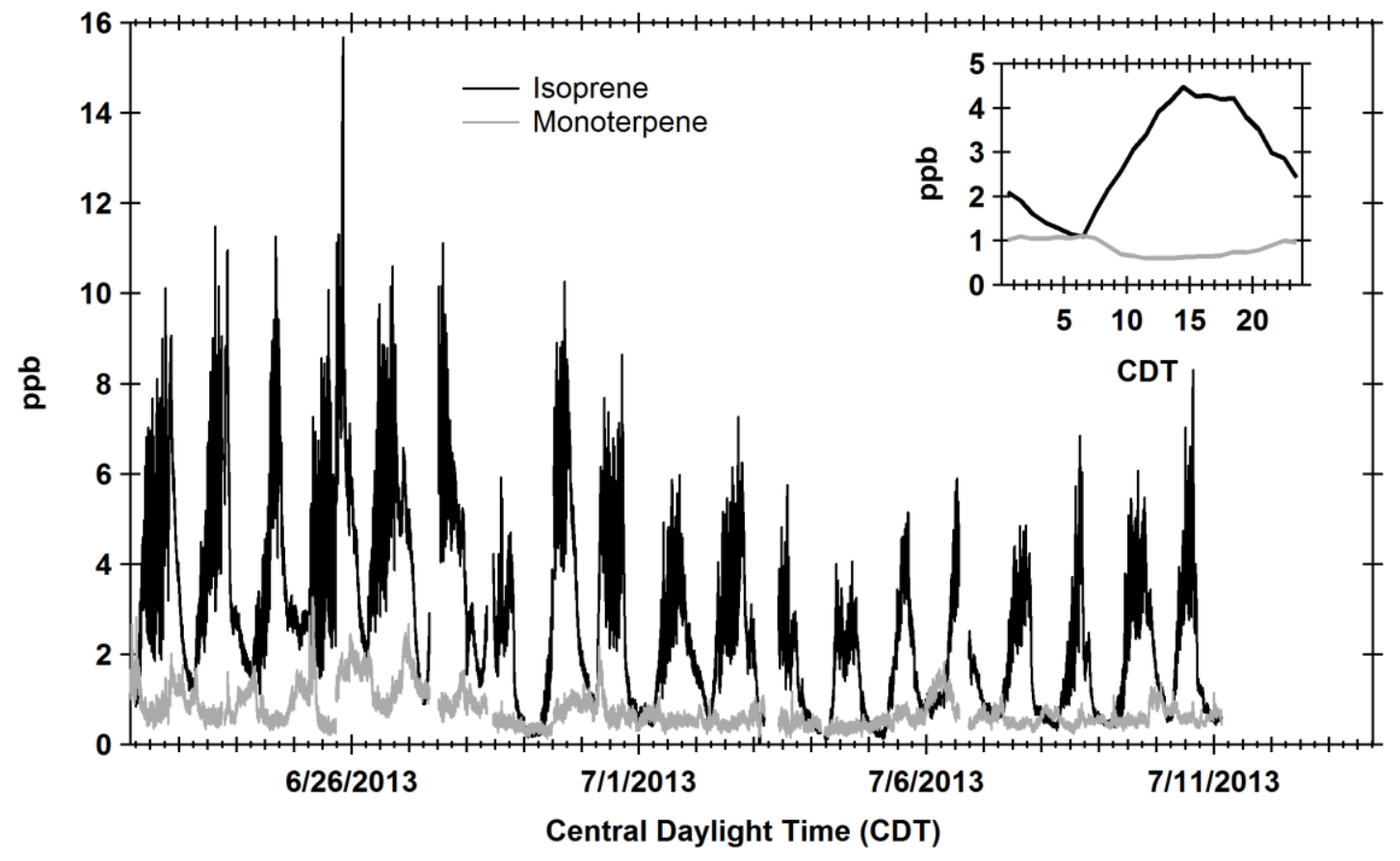

220 Figure S11: Time series of the monoterpene and isoprene signal (ppb) as measured by PTR-TOF-MS 221 along with their diurnal cycles (insert). The monoterpene had small diurnal oscillations and were 222 higher at nighttime while the isoprene signal reached higher daytime values, with a peak at 15:00. The 223 campaign average mass loadings of monoterpene and isoprene precursors were 0.85 and $2.8 \mathrm{ppb}$, 224 respectively. 
234 (1) Huey, L. G.; Hanson, D. R.; Howard, C. J. Reactions of $\mathrm{SF}_{6}$ - and I- with atmospheric trace gases. 235 J. Phys. Chem. 1995, 99(14), 5001-5008, DOI: 10.1021/j100014a021.

236 (2) Heinritzi, M.; Simon, M.; Steiner, G.; Wagner, A. C.; Kürten, A.; Hansel, A.; Curtius, J. 237 Characterization of the mass-dependent transmission efficiency of a CIMS. Atmos. Meas. Tech. 2016, 238 9, 1449-1460, DOI: 10.5194/amt-9-1449-2016.

239 (3) Yan, C.; Nie, W.; Äijälä, M.; Rissanen, M. P.; Canagaratna, M. R.; Massoli, P.; Junninen, H.; 240 Jokinen, T.; Sarnela, N.; Häme, S. A. K.; Schobesberger, S.; Canonaco, F.; Yao, L.; Prévôt, A. S. H.; 241 Petäjä, T.; Kulmala, M.; Sipilä, M.; Worsnop, D. R.; Ehn, M. Source characterization of highly 242 oxidized multifunctional compounds in a boreal forest environment using positive matrix 243 factorization. Atmos. Chem. Phys. 2016, 16, 12715-12731, DOI: 10.5194/acp-16-12715-2016.

244 (4) Cubison, M.J., Jimenez, J.-L. Statistical precision of the intensities retrieved from constrained 245 fitting of overlapping peaks in high-resolution mass spectra. Atmos. Meas. Tech. 2015, 8, 2333-2345, 246 DOI: 10.5194/amt-8-2333-2015.

247 (5) Ulbrich, I. M.; Canagaratna, M. R.; Zhang, Q.; Worsnop, D. R.; and Jimenez, J. -L. Interpretation 248 of organic components from Positive Matrix Factorization of aerosol mass spectrometric data. Atmos. 249 Chem. Phys. 2009, 9, 2891-2918, DOI: 10.5194/acp-9-2891-2009.

250 (6) Paatero, P. Least squares formulation of robust non-negative factor analysis. Chemometr. Intell. $251 \quad L a b .1997,37,23-35$.

252 (7) Paatero, P.; Hopke, P.K. Discarding or downweighting high-noise variables in factor analytic 253 models. Anal. Chim. Acta. 2003, 490,277-289, DOI: 10.1016/s0003-2670(02)01643-4. 